\title{
ON A TYPE OF SUBGROUPS OF A COMPACT LIE GROUP
}

\author{
YOZÔ MATSUSHIMA
}

Let $G$ be a connected compact Lie group and $H$ a connected closed subgroup. Then $H$ is an orientable submanifold of $G$ and we may consider $H$ as a cycle in $G$. In his interesting paper on the topology of group manifolds ${ }^{1)} \mathrm{H}$. Samelson has proved that, if $H$ is not homologous to 0 , then the homology ring?) of the coset space $G / H$ is isomorphic to the homology ring of a product space of odd dimensional spheres and the homology ring of $G$ is isomorphic to that of the product of the spaces $H$ and $G / H$. On the other hand, in a recent investigation of fibre bundles ${ }^{3)} T$. Kudo has shown that, if the homology ring of the coset space $G / H$ is isomorphic to that of an odd dimensional sphere, then $H$ is not homologous to 0 .

In the present paper we shall consider those connected closed subgroups of a connected compact Lie group $G$ such that the homology rings of the coset spaces are isomorphic to that of odd dimensional spheres. We shall first show that the problem to find all such subgroups of $G$ may be reduced to the case where $G$ is a simple group. The determination of such subgroups of the rotation groups of spheres (simple Lie groups of types $B$ and $D$ ) is contained essentially in a paper by $\mathrm{D}$. Montgomery and $\mathrm{H}$. Samelson on the transformation groups of spheres." Hence we shall consider here the above problem for simple Lie groups of the other types. The writer is grateful to Mr. M. Kuranishi for his friendly cooperation during the preparation of this paper.

I.

1. All groups considered in the following are compact Lie groups and sub-

Received July 29, 1950.

1) H. Samelson, Beiträge zur Topologie der Gruppen-Mannigfaltigkeiten, Ann. of Math Vol. 42 (1941); Satz VI. We refer to this paper as [S].

2) The coefficients of the homology ring are rational numbers.

3) T. Kudo, On the homological properties of fibre bundles, forthcoming in Journ. of the Institute of Polytechnics, Osaka City University.

4) D. Montgomery and H. Samelson, Transformation groups of spheres, Ann. of Math. Vol. 44 (1943). We refer to this paper as [M-S]. 
groups are always taken as closed.

a) The homology ring ${ }^{21}$ of an orientable manifold $M$ is denoted by $R(\dot{M})$ and $S^{n}$ denotes the $n$-sphere. The homology ring $R(G)$ of a compact Lie group $G$ is isomorphic to $R\left(S^{m_{1}} \times \ldots \times S^{m_{r}}\right)$, where $m_{i}$ are odd and $r$ is the rank $r(G)$ of $G^{5 \prime}$

b) A connected subgroup $H$ of a compact connected Lie group $G$ is said to be an $S$-subgroup, if $R(G / H)$ is isomorphic to $R\left(S^{m}\right)$, where $m$ is odd. If $H$ is an $S$-subgroup, then $r(H)=r(G)-10^{\text {) }}$

c) Let $G_{1}, \ldots, G_{k}$ be (compact connected) Lie groups and let $N$ be a finite normal subgroup of $\bar{G}=G_{1} \times \ldots \times G_{k}$. We say that the factor group $G=\bar{G} / N$ is essentially the product of $G_{1}, \ldots, G_{k}$ and we denote $G=G_{1} \circ \ldots \circ G_{k}$. Every compact connected Lie group $G$ is essentially the producted of some simply connected simple groups and a toral group. If $G_{1}$ is a connected normal subgroup of a compact connected Lie group $G$, then there exists a connected normal subgroup $G_{2}$ of $G$ such that $G=G_{1} \circ G_{2} .^{7}$ )

d) Let $G$ be a Lie group and $H$ a subgroup and let $W=G / H$. Then we may consider $G$ in a natural way as a transitive transformation group of $W$. The set of all elements $g \in G$ for which $g(x), x \in W$, are identity transformation of $W$ form a normal subgroup $G_{0}$ contained in $H$. If $G_{0}$ is a finite group, then $G$ is said to be almost effective on $W$.

2. We prove now a theorem on the structure of $S$-subgroups. Let $R_{1}$ be the rotation group of 1 -sphere and $\tilde{R}_{2}$ the simply connected covering group of the rotation group $R_{2}$ of 2 -sphere.

THEOREM I. Let $G$ be a compact connected Lie group, $H$ an S-subgroup of $G$, and let $G_{2}$ be the maximal connected normal subgroup of $G$ contained in $H$. Further, let $G_{1}$ be a connected normal subgroup of $G$ such that $G=G_{1} \circ G_{2}$. Then $H=H_{1} \circ G_{2}$ and $H_{1}$ is an S-subgroups of $G_{1}$ and $G_{1}$ is simple or essentially the product of two simple groups one of which is $R_{1}$ or $\tilde{R}_{\mathbf{2}}$.

3. To prove Theorem I we need some lemmas.

Lemma 1. If $H$ is a connected normal subgroup of $G$, then $H+0$.

Proof. Let $K$ be a connected normal subgroup of $G$ such that $G=H \circ K$

5) H. Hopf, Über die Topologie der Gruppen-Mannigfaltigkeiten und ihre Verallgemeinerungen, Ann. of Math. Vol. 42 (1941) and H. Hopf, Über den Rang geschlossener Lieschen Gruppen, Commet. Math. Helvet. Vol. 13 (1941).

6) See, [S], Satz VI. Note that by the results of H. Samelson and T. Kudo $H$ is an $S$ subgroup if and only if $H$ is not homologous to 0 and $r(H)=r(G)-1$.

7) In this case we may consider $G_{1} \circ G_{2}$ as the usual product of two normal subgroups $G_{1}$ and $G_{2}$. 
and let $\bar{G}=H \times K$. Then $H$ is obviously +0 in $\bar{G}$. But the natural homomorphism $\pi$ of $\bar{G}$ onto $G$ induces the isomorphic mapping of the homology group $B(\bar{G})$ of $\bar{G}$ onto the homology group $B(G)$ of $G$. From these facts we conclude without difficulty that $H+0$ in $G$.

Lemia 2. Let $G=G_{1} \circ G_{2}, \bar{G}=G_{1} \times G_{2}$ and let $\pi$ be the natural homomorphic mapping of $\bar{G}$ onto $G$. Further let $H$ be a connected subgroup of $G$ and $\bar{H}$ the connected component containing the identity of the group $\pi^{-1}(H) . H$ is an $S$-subgroun of $G$ if and only if $\bar{H}$ is an $S$-subgroup of $\bar{G}$, and $R(G / H)$ $\cong R(\bar{G} / \bar{H})$.

Proof. $\pi$ induces the isomorphic mapping of the homology group $B(\bar{G})$ of $\bar{G}$ onto the homology group $B(G)$ of $G$. The same holds for $B(\bar{H})$ and $B(H)$, since $\pi(\bar{H})=H$ and $\pi$ is locally isomorphic. Let $V(\bar{H})$ be the additive subgroup of $B(\bar{H})$ composed of all minimal elements of $B(\bar{H})^{\text {s) }}$ and let $\bar{v}_{1}, \ldots, \bar{v}_{k}$ be a basis of $V(\bar{H})$. Then $\pi\left(\bar{v}_{1}\right), \ldots, \pi\left(\bar{v}_{k}\right)$ is also a basis of the group $V(H)$ of the minimal elements of $B(H) .^{s)}$ Assume $H+0$. Then $\pi\left(\bar{v}_{1}\right), \ldots, \pi\left(\bar{v}_{k}\right)$ are linearly independent also when we consider $\pi\left(\bar{v}_{i}\right)$ as homology classes in G. ${ }^{9)}$ If $\bar{H} \sim 0$, then $\bar{v}_{1}, \ldots, \bar{v}_{k}$ would be linearly dependent considered as homology classes in $\bar{G}$, and the same for $\pi\left(\bar{v}_{3}\right), \ldots, \pi\left(\bar{v}_{k}\right)$ considered as the homology class in $G$. Hence $\bar{H}+0$. Conversely, if $\bar{H}+0$, then $H+0$. Since $r(H)=r(\bar{H}), r(G)=r(\bar{G})$ and $r(H)=r(G)-1$, we have $r(\bar{H})=r(\bar{G})-1$. Hence $R(\bar{G} / \bar{H}) \cong R\left(S^{m}\right)$. Further since $\operatorname{dim} G / H=\operatorname{dim} \bar{G} / \bar{H}$ we have clearly $R(G / H) \cong R\left(S^{m}\right)$.

4. Proof of Theorem $I^{10 ;}$ Let $\bar{G}=G_{1} \times G_{2}, \pi$ be the natural homomorphism of $\bar{G}$ onto $G$ and let $\bar{H}$ be the connected component of the group $\pi^{-1}(H)$. Since $\bar{H} \supset G_{2}$, we have $\bar{H}=H_{1} \times G_{2}$, where $H_{1}$ is a connected subgroup of $G_{1}$. By Lemma $2 \bar{H}+0$ in $\bar{G}$, hence $H_{1}+0$ in $G_{1}$ and $H_{1}$ is clearly an $S$-subgroup of $G_{1}$ containing no connected normal subgroup of $G_{1}$ (different from the group consisting only of identity of $G_{1}$ ). We shall prove that $G_{1}$ has the structure stated in Theorem I. For simplicity we write $G$ and $H$ in place of $G_{1}$ and $H_{1}$. Then $F$ is an $S$-subgroup of $G$ containing no connected normal subgroup of $G$. Let $W=G / H$ and $R(W)=R\left(S^{m}\right)$ ( $m$ being odd). Then $G$ is almost effective on $W$. If $G=G_{1} \circ G_{2}$, then we show that one of $G_{i}$, say $G_{1}$, is transitive on $W$. For this purpose let $\bar{G}=G_{1} \times G_{2}$, let, as above, $\bar{H}$ be the connected component

s) For the definition and the properties of the minimal element, see H. Hopf, loc. oit. and $[\mathrm{S}]$.

9) See, [S], Satz III. Korollar 1.

10) The following proof is similar to the proof of Theorem $I$ b) in [M-S]. But we avoid to use a theorem of Gysin which played an essential role there. 
of $\pi^{-1}(H)$ and $\bar{W}=\bar{G} / \bar{H}$. Then $R(\bar{W})=R\left(\mathrm{~S}^{m}\right)$ and $\bar{G}$ is also almost effective on $\bar{W}$. If we can show that $G_{1}$ is transitive on $\bar{W}$, then we see easily that $G_{1}$ is also transitive on $W$. Let $\Gamma_{i}$ be the image of $\bar{H}$ under the natural homomorphism $g_{1} \cdot g_{2} \rightarrow g_{i}$ of $\bar{G}$ onto $G_{i}$ and let $\Gamma=\Gamma_{1} \times \Gamma_{\mathrm{g}} . \quad \Gamma_{i}$ are connected and $\Gamma \supseteqq H$. Let $H_{i}$ be the intersections $\bar{H} \cap G_{i} . H_{i}$ are normal subgroups of $\Gamma_{i}$. Then, as in the proof of Theorem I b) in [M-S], the spaces $\Gamma / \bar{H}, \Gamma_{1} / H_{1}, \Gamma_{2} / H_{2}$ are homeomorphic. Consider the space $\Gamma_{1} / H_{3}$. As $H_{1}$ is a normal subgroup of $\Gamma_{1}$, it is a compact connected Lie group. If $\Gamma_{1}=H_{1}$, then $\Gamma_{2}=H_{2}$. If follows that $\bar{H}=H_{1} \times H_{2}$ and $\bar{G} / \bar{H}=G_{1} / H_{1} \times G_{2} \times H_{2}$. But since $R(\bar{G} / \bar{H})=R\left(S^{m}\right)$, the space $\bar{G} / \bar{H}$ can not be decomposed into a direct product of two manifolds of positive dimensiens. Hence one of the spaces $G_{i} / H_{i}$, for example $G_{2} / H_{2}$, must be a point. This means that $G_{2}=H_{2}$ and hence $\bar{H}$ must contain the normal subgroup $G_{2}$, which is impossible. Hence $\Gamma_{1} \neq H_{1}$. Since $r(\bar{H})=r(\bar{G})-1$, we can show that $r\left(\Gamma_{1} / H_{1}\right)=1$. Therefore $\Gamma_{1} / H_{1}$ is homeomorphic with one of the three following manifolds : the 1-sphere $S^{1}$, the 3 -sphere $S^{3}$, and the projective 3 -space $P^{3}$. We shall show that one of $\Gamma_{i}$ is equal to $G_{i}$.

i) First let $\Gamma / \bar{H} \approx \Gamma_{i} / H_{i} \approx S^{3}$.

Since $S^{3}$ is simply connected, $H_{i}$ are connected. Clearly $r(\bar{G})=r(\Gamma)$ and $r(\bar{H})=r(\bar{G})-1$. As $H_{i}$ are the normal subgroups of $\bar{H}, H_{i}+0$ in $\bar{H}$ by Lemma 1. Since $\bar{H}+0$ in $\bar{G}$, it follows that $H_{i}+0$ in $\bar{G}$, "') whence $H_{i}+0$ in $G_{i}$. Let $\Delta=H_{1} \times H_{2}$. Then $\Delta+0$ in $\bar{G}$ and since $r\left(H_{i}\right)=r\left(\Gamma_{i}\right)-1, r(\Delta)=r(\bar{H})-1$. Hence $\Delta$ is an $S$-subgroup of $\bar{H}$. Then, from the relations $R(\bar{G})=R(\bar{G} / \bar{H} \times \bar{H})$ and $R(\bar{H})=R(\bar{H} / \Delta \times \Delta)$, ${ }^{\text {'2) }}$ we obtain $R(\bar{G})=R(\bar{G} / \bar{H} \times \bar{H} / \Delta \times \Delta)$. But since $R(\bar{G})=R(\bar{G} / \Delta \times \Delta)$, it follows that $R(\bar{G} / \Delta)=R\left(G_{1} / H_{1} \times G_{2} / H_{2}\right)=R(\bar{G} / \bar{H}$ $\times \bar{H} / \Delta)$. However, $\bar{H}$ and $\Delta$ are $S$-subgroups of $\bar{G}$ and $\bar{H}$ respectively, and therefore $R(\bar{G} / \bar{H})=R\left(S^{m}\right)$ and $R(\bar{H} / \Delta)=R\left(S^{k}\right)$ ( $m$ and $k$ being odd). Hence $R\left(G_{1} / H_{3}\right.$ $\left.\times G_{2} / H_{2}\right)=R\left(S^{m} \times S^{k}\right)$. Then it follows that $R\left(G_{1} / H_{1}\right)=R\left(S^{m}\right)$ and $R\left(G_{2} / H_{2}\right)$ $=R\left(S^{k}\right)$. On the other hand $R\left(G_{2}\right)=R\left(H_{2} \times G_{2} / H_{2}\right)=R\left(H_{2} \times S^{k}\right)$ and $R\left(\Gamma_{2}\right)$ $=R\left(H_{2} \times \Gamma_{2} / H_{2}\right)=R\left(H_{2} \times S^{3}\right)$. But since $\operatorname{dim} \Gamma-\operatorname{dim} \Delta=6$ and $\operatorname{dim} \Gamma-\operatorname{dim}$ $\bar{H}=3$, we have $\operatorname{dim} \bar{H}-\operatorname{dim} \Delta=3$. Hence $k=3$. This shows that $\operatorname{dim} G_{2}$ $=\operatorname{dim} \Gamma_{2}$ and hence $G_{2}=\Gamma_{2}$.

ii) Next let $\Gamma / \bar{H} \approx \Gamma_{i} / H_{i} \approx P^{3}$.

In this case $H_{i}$ need not be connected. But if $H_{i}{ }^{0}$ are the connected components of $H_{i}$, then $\Gamma_{i} / H_{i}^{0}$ are the covering spaces of $P^{3}$, whence homeomorphic to $S^{3}$. So, replacing $H_{i}$ by $H_{i}^{0}$, if necessary, we obtain $G_{2}=\Gamma_{2}$ by the same argument as in i).

11) See, [S], Satz III Korollar 3.

19) See, [S], Satz VI. 
iii) Finally let $\Gamma / \bar{H} \approx \Gamma_{i} / H_{i} \approx S^{1}$.

Let $H_{i}{ }^{0}$ be the connected components of $H_{i}$. Then $\Gamma_{i} / H_{i}{ }^{0}$ are also homeomorphic to $S^{1}$. Hence we may assume that $H_{i}$ are connected. In this case $\bar{H} / \Delta=S^{1}$ and by the same argument as in i), we have $R\left(G_{3} / H_{3} \times G_{2} / H_{2}\right)$ $=R(\bar{G} / \bar{H} \times \bar{H} / \Delta)=R\left(S^{m} \times S^{1}\right)$. Hence $R\left(G_{1} / H_{1}\right)=R\left(S^{m}\right)$ and $R\left(G_{2} / H_{2}\right)$ $=R\left(S^{1}\right)$. It follows that $\operatorname{dim} G_{2}-\operatorname{dim} H_{2}=\operatorname{dim} \Gamma_{2}-\operatorname{dim} H_{2}=1$. Hence $G_{2}=\Gamma_{2}$.

Thus we have proved that $G_{2}=\Gamma_{2}$. Then we may show as in [M-S] that $G_{1}$ is transitive on $W$ and that $r\left(G_{2}\right)=1$ i.e. $G_{2}$ is $R_{1}$ or $R_{2}$ or $\tilde{R}_{2}$. Theorem I will be proved, if we show that $G_{1}$ is simple. Since $G_{1}$ is transitive on $W$, there exists a subgroup $H_{1}$ of $G_{1}$ such that $W=\mathrm{G}_{1} / H_{1}$, where $H_{1}=G_{1} \cap H$. Let $H_{1}{ }^{0}$ be the connected component of $H_{1}$. Then $H_{1}{ }^{0}$ is a normal subgroup of $H$. Hence $H_{1}^{0}+0$ in $H$. Then, by the same argument as above, $H_{1}^{0}+0$ in $G_{1}$. Moreover, we may easily verify that $r\left(H_{1}^{0}\right)=r\left(G_{1}\right)-1$, whence $H_{1}^{0}$ is an $S$ subgroup of $G_{1}$ and $R\left(W_{1}\right)=R\left(S^{m}\right)$. Clearly $H_{1}^{0}$ contains no connected normal subgroup of $G_{1}$ different from the identity. Suppose that $G_{1}$ is not simple and let $G_{1}=G^{\prime} \circ G^{\prime \prime}$. We use for $G_{1}, H_{1}^{0}$ and $W_{1}$ the same argument we used for $G, H$ and $W$ and find that $G^{\prime}$ is transitive on $W_{1}$ and $G^{\prime \prime}$ is $R_{1}$ or $R_{2}$ or $\widetilde{R}_{2}$. Then $\boldsymbol{G}=G^{\prime} \circ\left(G^{\prime \prime} \circ G_{2}\right)$. Since the rank of the group $G_{3}=G^{\prime \prime} \circ G_{2}$ is 2 , it must be transitive on $W$. By the same argument as above, there exists in $G_{3}$ an $S$ subgroup $H_{3}$ such that $R\left(G_{3} / H_{3}\right)=R\left(S^{m}\right)$ and $H_{3}$ contains no connected normal subgroup of $G_{3}$. Then $G^{\prime \prime}$ or $G_{2}$ must be transitive on $G_{3} / H_{3}$. This is impossible if $m>3$. The cases $m=1$ and 3 may be treated easily for themselves. Thus Theorem $I$ is proved.

5. By Theorem I the problem to find all $S$-subgroups of a compact connected group $G$ is reduced to the cases where $G$ is a simple group or a direct product of two simple groups one of which is of rank 1. The latter case may be reduced to the former case. So we consider in the following the $S$-subgroups of a simple group.

\section{II.}

1. If $G$ and $G^{\prime}$ are locally isomorphic compact simple groups, then, as we may easily see from Lemma 2 , the $S$-subgroups of $G$ and $G^{\prime}$ correspond to each other. Hence we have only to consider one respresentative from each class of locally isomorphic groups. In particular, if we can show that the S-groups of a simple group $G$ are corjugate to each other, then the same folds for every simple group locally isomorphic to $G$.

2. First we consider the case $G=R_{n}$, the rotation group of $n$-sphere. We denote by $Q_{n-k}$ the subgroup of $R_{n}$ composed of all elements of $R_{n}$ which leave 
fixed the unit point on the first $k$ of $n+1$ axis of Euclidean $(n+1)$-space $E_{n+1}$. Clearly $Q_{n-k}$ is isomorphic to $R_{n-k}$.

i) $n:=2 m-1$.

Then $R_{n} / Q_{n-1}=S^{2 m-1}$ and hence $Q_{n-1}+0$ in $R_{n}{ }^{13)}$ By Lemma 7 of [M-S] we see that every $S$-subgroup of $R$ is conjugate to $Q_{n-1}$ except for a finite number of $n$ 's.

ii) $n=2 m$.

In this case $Q_{n-2}+0$ in $R_{n}$. By the proof of Theorem IV of [M-S] we see that every $S$-subgroup of $R_{n}$ is conjugate to $Q_{n-2}$ except for a finite number of $n$ 's.

\section{III.}

1. Here we consider the group $G=A_{n}$, the unimodular unitary group in $n+1$ variables. We denote by $A_{n-1}$ the subgroup of $G=A_{n}$ consisting of all elements of $G$ which leave fixed the unit point on the first of the $n+1$ axis of unitary $(n+1)$-space. Then $G / A_{n-1}=S^{2 n+1}$, whence $A_{n-1}+0 . .^{13)}$

We prove the following

Theorem II. Every S-subgroup of $G=A_{n}$ is conjugate to $A_{n-1}$ for $n \geqslant 8$. 14)

2. Let $U$ be an $S$-subgroup of $G$. Then $R(G / U)=R\left(S^{m}\right)$ ( $m$ : odd) and $R(G)=R(G / U \times U)$. Hence $R(G)=R\left(U \times S^{m}\right)$. The homology ring of $G$ is

$$
\left.R(G)=R\left(A_{n}\right)=R\left(S^{3} \times S^{i} \times \ldots \times S^{2 n+1}\right) .^{15}\right)
$$

Hence $m=2 k+1$ and

$$
R(U)=R\left(S^{3} \times \mathrm{S}^{5} \times \ldots \times S^{m-2} \times S^{m+2} \times \ldots \times S^{2 n+1}\right) .
$$

$U$ is simple, for in (1) $S^{3}$ appears only once. ${ }^{16)}$ As we may easily verify the group $A_{n}$ can not contain the exceptional groups of rank $n-1$. Hence $U$ is a classical simple group. Then (1) is possible for $m<2 n+1$ only when $n=3$, $m=5$, and $R(G)=R\left(S^{3} \times S^{5} \times S^{7}\right)$ and $R(U)=R\left(S^{3} \times S^{7}\right)$. Hence if $n>3$ then $m=2 n+1$ and $R(U)=R\left(S^{3} \times S^{5} \times \ldots \times S^{2 n-1}\right)$. This shows that $U$ is a simple group of type $A_{\left.n-1 \cdot 0^{15}\right)}$

13) See, [S], Satz IV.

14) The writer can not decide whether Theorem II is also valid for $n<8$ or not. Since every subgroups of rank 1 is not homologous to 0 , Theorem II is not valid for $\boldsymbol{n}=\mathbf{2}$ as we may show by an example. Cf. J. L. Koszul, C. R. Paris 225, p. 477 (1947), and H. Samelson, C. R, Paris 228, p. 630 (1949).

15) See, L. Pontrjagin, Homologies in compact Lie groups, Rec. Math. N. S. Vol. 6 (1939) or [S].

16) For, by a theorem of E. Cartan, the 3-dimensional Betti number of any semi-simple group is not equal to 0 . 
3. Let $\mathfrak{G}_{c}$ be the Lie algebra of $G$ and $\mathfrak{H}_{c}$ the subalgebra of $\mathscr{B S}_{c}$ corresponding to the subgroup $U$. By taking a suitable conjugate group of $U$, we may assume that a maximal abelian subalgebra $\mathfrak{h}_{c}$ of $\mathfrak{H}_{c}$ is contained in a fixed maximal abelian subalgebra $\mathscr{S}_{c}$ of $\mathscr{G}_{c^{\prime}}{ }^{i n}$ We denote by $(\mathbb{S}$ and $\mathfrak{U}$ the Lie algebras obtained from $\mathfrak{G}_{c}$ and $\mathfrak{H}_{c}$ respectively by extending the domain of coefficients to the complex number field $K$ 。 $\mathfrak{S}$ and $h$ may be defined analogously. Then as is well known, $B$ is the Lie algebra consisting of all matrices of degree $n+1$ with complex numbers as coefficients whose traces are 0 . Let $e_{i k}(i, k=1, \ldots$, $n+1)$ be the matrix whose $(i, k)$-element is 1 and others are all 0 and $h_{i}=e_{i}$. Then $\&$ has the following basis:

$$
\begin{gathered}
\mathscr{B}=\mathfrak{S}+\sum_{i, k=1}^{n+1} K e_{i k},(i \neq k) ; \mathfrak{S}=\left\{\lambda^{1} h_{1}+\ldots+\lambda^{n+1} h_{n+1}\right\}, \sum_{i=1}^{n+1} \lambda^{i}=0 ; \\
{\left[\sum_{j=1}^{n+1} \lambda^{j} h_{j}, e_{i k}\right]=\left(\lambda^{i}-\lambda^{k}\right) e_{i k} .}
\end{gathered}
$$

The real Lie algebra $\mathbb{S}_{c}$ is obtained from $B$ by the so called "unitary restriction." Since $\mathbb{U}$ is of type $A_{n-1}, \mathfrak{U}$ has the following basis :

$$
\mathfrak{U}=K \widetilde{h}_{1}^{\prime}+\ldots+K \tilde{h}_{n-1}^{\prime}+\sum_{i, k=1}^{n} K u_{i k},(i \neq k),
$$

where $\tilde{h}_{1}^{\prime}, \ldots, \tilde{h}_{n+1}^{\prime}$ is a linearly independent basis of $h$ and

$$
\begin{aligned}
& {\left[\sum_{j=1}^{n-1} \mu^{j} \widetilde{h}_{j}^{\prime}, u_{i k}\right]=\left(\mu^{i}-\mu^{k}\right) u_{i k},(i, k \leqq n-1) ;} \\
& {\left[\sum_{j=1}^{n-1} \mu^{j} \widetilde{h}_{j}^{\prime}, u_{i n}\right]=\left(\mu^{i}+\mu^{1}+\ldots+\mu^{n-1}\right) u_{i n},} \\
& {\left[\sum_{j=1}^{n-1} \mu^{j} \widetilde{h}_{j}, u_{i n}\right]=\left(-\mu^{i}-\mu^{1}-\ldots-\mu^{n-1}\right) u_{n i} .}
\end{aligned}
$$

Now, let $\widetilde{h}_{n}$ be an element of $\mathscr{S}_{2}$ which is not contained in $\mathfrak{h}$ and let $\widetilde{h}_{i}=\widetilde{h}_{i}{ }^{\prime}+\widetilde{h}_{n}$ for $i=1, \ldots, n-1$. Then $\mu^{1} \widetilde{h}_{1}^{\prime}+\ldots+\mu^{n-1} \widetilde{h}_{n-1}^{\prime}=\mu^{1} \widetilde{h}_{1}+\ldots+\mu^{n} \widetilde{h}_{n}$ and $\mu^{1}+\ldots+\mu^{n}=0$. Hence $\mathfrak{h}$ is the set of all elements $\lambda^{1} \tilde{h}_{1}+\ldots+\lambda^{n} \tilde{h}_{n}$ such that $\sum_{i=1}^{n} \lambda^{i}=0$. We have

$$
\left[\sum_{j=1}^{n} \lambda^{j} \tilde{h}_{j}, u_{i k}\right]=\left(\lambda^{i}-\lambda^{k}\right) u_{i k}
$$

17) For, any toral sutgroup of a compact connected Lie group $G$ is conjugate to a subgroup of any maximal toral subgroup $G$. See, A. Weil, Démonstration topologique d'un théorème fondamental de Cartan. C. R. Paris 200 (1935) ; H. Hopf and H. Samelson, Ein Satz über die Wirkungsräume geschlossener Liescher Gruppen, Commet. Math. Helvet. Vol. 13 (1941). 
for $1 \leqq i, k \leqq n$.

Clearly $u_{i k}$ 's are linear combinations of $e_{r s}$ 's. A $u_{i k}$ is said to be singular if it is a linear combination of at least two $e_{r s}$ 's. We shall prove that no $u_{i k}$ is singular for $n \geqq 8$.

4. Let $\tilde{h}_{i}=\sum_{k=1}^{n+1} \mu_{i}^{k} h_{k}$, where $\sum_{k=1}^{n+1} \mu_{i}^{k}=0$. Since $\tilde{h}_{1}, \ldots, \tilde{h}_{n}$ are linearly independent elements of $\mathfrak{g}$, det. $\left(\mu_{i}^{k}\right)_{i, k=1}, \ldots, n \neq 0$. Let $\mu^{i}=\left(\mu_{1}^{i}, \ldots, \mu_{n}^{i}\right)$ for $i=1, \ldots, n+1$. Then the vectors $\mu^{1}, \ldots, \mu^{n}$ are linearly independent and $\mu^{n+1}=-\mu^{1}-\ldots-\mu^{n}$. Hence $n$ vectors taken from $\mu^{1}, \ldots, \mu^{n+1}$ are all linearly independent. Now $\left[\sum_{j=1}^{n} \lambda^{j} \tilde{h}_{j}, e_{i k}\right]=\left(\sum_{j=1}^{n} \lambda^{j}\left(\mu_{j}{ }^{j}-\mu_{j}^{k}\right)\right) e_{i k}$ and $\left[\sum_{j=1}^{n} \lambda^{j} \tilde{h}_{j}, e_{r s}\right]$ $=\left(\sum_{j=1}^{n} \lambda^{j}\left(\mu_{j}^{r}-\mu_{j}^{s}\right)\right) e_{r s}$. If $\sum_{j=1}^{n} \lambda^{j}\left(\mu_{j}^{i}-\mu_{j}^{k}\right)=\sum_{j=1}^{n} \lambda^{j}\left(\mu_{j}^{r}-\mu_{j}^{s}\right)$ for every $\lambda^{j}$ such that $\sum_{j=1}^{n} \lambda^{j}=0$, then, as we may easily verify, $\mu^{i}-\mu^{k}=\mu^{r}-\mu^{s}+\xi\left(\begin{array}{l}r s \\ i k\end{array}\right)$, where $\xi\left(\begin{array}{c}r s \\ i k\end{array}\right)=(c, \ldots, c)$. If this relation holds, we define $e_{i k} \equiv e_{r \dot{s}}$. We may easily see that if $e_{i k}$ and $e_{r s}$ appear in a singular $u_{j l}$ then $e_{i k} \equiv e_{r s}$.

5. First we consider the case where a relation $\mu^{i}-\mu^{k}=\xi$ holds for some $i, k$, where $\xi$ is a vector whose components are all equal, i.e. $\xi=(d, \therefore, d){ }^{18)}$ Then $\left[\sum_{j=1}^{n} \lambda^{j} \tilde{h}_{j}, e_{i k}\right]=\left(\sum_{j=1}^{n} \lambda^{j}\left(\mu_{j}^{i}-\mu_{j}{ }^{k}\right)\right) e_{i k}=\left(\sum_{j=1}^{n} \lambda^{j} d\right) e_{i k}=0$.

Since $\mathfrak{h}$ is $(n-1)$-dimensional, we may easily see that $\mathfrak{h}$ is the subspace of $\$$ consisting of all elements $\lambda^{1} h_{1}+\ldots+\lambda^{n+1} h_{n+1}$ such that $\lambda^{j}=\lambda^{k}$. For simplicity, let $i=n, k=n+1$. Let $\left[h, e_{i k}\right]=\alpha e_{i k}$ and $\left[h, e_{r s}\right]=\beta e_{r s}$, where $h \in \mathfrak{h}$. Then $e_{i k} \equiv e_{r s}$, if and only if $\alpha=\beta$ for every $h \in \mathfrak{h}$. Hence in our case, $e_{i k} \equiv e_{r s}$ if and only if $\lambda^{i}-\lambda^{k}=\lambda^{r}-\lambda^{s}$. As we may easily verify, the followings are possible :

$$
e_{n, k} \equiv e_{n+1, k}, e_{k, n} \equiv e_{k, n+1},(k<n), e_{n, n+1} \equiv e_{n+1, n} .
$$

Since $\left[h, e_{n, n+1}\right]=\left[h, e_{n+1}, n\right]=0$ holds for every $h \in \mathfrak{h}, e_{n, n+1}$ and $e_{n+1, n}$ can not appear in $u$ 's as factors. Hence if some $u$ 's are singular, these $u$ 's must be the form $u_{\alpha}=a e_{n, k}+b e_{n+1, k}, u_{\beta}=c e_{k, n}+d e_{k, n+1}$ and the "roots" $\alpha$ and $\beta$ corresponding to these $u$ 's satisfy the relation $\alpha=-\beta$. Hence $\left[u_{\alpha}, u_{\beta}\right]=\left[a e_{n, k}\right.$ $\left.+b e_{n+1, k}, c e_{k, n}+d e_{k, n+1}\right]=a c\left(h_{n}-h_{k}\right)+b d\left(h_{n+1}-h_{k}\right)+b c e_{n+1, n}+a d e_{n, n+1}$ $\in \mathfrak{h}$. Hence $b c=a d=0$ and $a c=b d$. This is clearly a contradiction and hence all $u$ 's are not singular.

6. Now suppose $e_{i k} \equiv e_{r s}$. Then $\mu^{i}-\mu^{k}=\mu^{r}-\mu^{s}+\xi\left(\begin{array}{c}r s \\ i k\end{array}\right)$. If $\xi\left(\begin{array}{c}r s \\ i k\end{array}\right)=0$.

18) Through in the following we denote by $\xi$ such a vector. 
then $e_{r s}=e_{i k}$ or $e_{r s}=e_{k i}$, since 4 vectors taken from $\mu^{1} \ldots \mu^{n+1}$ are linearly independent for $n \geqslant 4$ (c.f. 4.) and $i \neq \dot{k}$ and $r \neq s$. If $e_{i k} \equiv e_{k i}$, then the relation $\mu^{i}-\mu^{k}=\xi$ holds and hence all $u^{\prime}$ s are not singular. Thus we may assume $\xi\left(\begin{array}{c}r s \\ i k\end{array}\right) \neq 0$. Further if $i=r$ or $k=s$, we also have the relations $\mu^{k}-\mu^{s}=\xi$ or $\mu^{i}-\mu^{r}=\xi$. Hence we may assume $i \neq r, k \neq s$.

7. Suppose that $e_{i k} \equiv e_{r s}$ and $e_{u v} \equiv e_{x y}$ holds. We may assume that $i \neq r$, $k \neq s, u \neq x$ and $v \neq y$. Then we have the relations $\mu^{i}-\mu^{k}=\mu^{r}-\mu^{s}+\xi\left(\begin{array}{c}r s \\ i k\end{array}\right)$ and $\mu^{u}-\mu^{v}=\mu^{x}-\mu^{y}+\xi\left(\begin{array}{c}x y \\ u v\end{array}\right)$. We may assume that $\xi$ 's are $\neq 0$ and $\xi\left(\begin{array}{c}r s \\ i k\end{array}\right)$ $=d \xi\left(\begin{array}{l}x y \\ u v\end{array}\right)$, where $d(\neq 0)$ is a complex number. Then we obtain the relation

$$
\mu^{i}-\mu^{k}-d \mu^{u}+d \mu^{v}=\mu^{r}-\mu^{s}-d \mu^{x}+d \mu^{y} .
$$

i) Let $d \neq \pm 1$. Since 8 vectors taken from $\mu^{1} \ldots \mu^{n+1}$ are linearly independent for $n \geqq 8$, we must have $\mu^{i}-\mu^{k}-\mu^{r}+\mu^{s}=0, \mu^{u}-\mu^{v}-\mu^{x}+\mu^{y}=0$ and moreover $i=s, k=r, u=y$ and $v=x$. Hence $e_{i k} \equiv e_{k i}$ and $e_{u v} \equiv e_{v u v}$ Then $\mu^{i}-\mu^{k}=\xi$ and $\mu^{u}-\mu^{v}=\xi$ hold and all $u^{\prime}$ s are not singular (c.f. 5 and 6).

ii) Let $d= \pm 1$. Then $\mu^{i} \pm \mu^{v}+\mu^{s} \pm \mu^{x}-\mu^{k} \mp \mu^{u}-\mu^{r} \mp \mu^{y}=0$. As in i) 8 vectors are linearly independent and since $i \neq k, r \neq s, u \neq v, x \neq y, i \neq r$, $k \neq s, u \neq x$ and $v \neq y$, we may verify that the following cases are possible:

$$
\begin{array}{ll}
e_{i k} \equiv e_{r s}, & e_{i r} \equiv e_{k s} \\
e_{i k} & \equiv e_{r s}, \quad e_{r i} \equiv e_{s k} \\
e_{i k} & \equiv e_{r s}, \quad e_{k i} \equiv e_{s r} \\
e_{i k} & \equiv e_{k i}, \quad e_{u v} \equiv e_{v u} \\
e_{i k} & \equiv e_{k s}, \quad e_{s v} \equiv e_{v i} \\
e_{i k} & \equiv e_{k s}, \quad e_{i v} \equiv e_{v s}
\end{array}
$$

But in the cases 4),5) and 6), we obtain the relation of the form $\mu^{l}-\mu^{m}=\xi$. Hence we have only to treat 1 ), 2) and 3 ).

8. Then it is easily verified that the possible singular $u$ 's are as follows :

$$
\begin{array}{ll}
u_{\alpha}=a_{\alpha} e_{i k}+b_{\alpha} e_{r s}, & u_{-\alpha}=a_{-\alpha} e_{k i}+b_{-\alpha} e_{s r} ; \\
u_{\beta}=a_{\beta} e_{i r}+b_{\beta} e_{k s}, & u_{-\beta}=a_{-\beta} e_{r i}+b_{-\beta} e_{s k} .
\end{array}
$$

Let $u_{\alpha}=u_{p q}$. Then $u_{\alpha}=\left[u_{p t}, u_{t q}\right](t=1, \ldots, n)$. Clearly, for some $t, u_{p t}$ and $u_{t q}$ are not singular. Let $u_{p t}=a e_{u v}$ and $u_{t q}=b e_{x y}$. Then $u_{\alpha}=\left[u_{p t}, u_{t q}\right]$ $=\left[a e_{u v}, b e_{x y}\right]=a b\left(\delta_{v x} e_{u y}-\delta_{u y} e_{x v}\right)$. Hence it follows that $u=i, y=k, x=r$, $v=s$ or $u=r, y=s, x=i, v=k$. Then, since $i \neq k$ and $r \neq s, \delta_{u y}=\delta_{v \cdot x}=0$. However, they can not bo the case. Thus we have proved that no $u$ is singular. 
9. Let $\imath_{\alpha}=a_{\alpha} e_{i_{\alpha} j_{\alpha}}$. We want to prove that the set of indices $S=\bigcup_{\alpha}\left\{i_{\alpha}, j_{\alpha}\right\}$ is a proper subset of $\{1,2, \ldots, n+1\}$. Suppose, for this purpose, that $S=\{1$, $2, \ldots, n+1\}$. Let $1 \leqq s, t \leqq n+1$. Then there exist $\alpha$ and $\beta$ such that $s=i_{\alpha}$ or $j_{\alpha}$ and $t=i_{\beta}$ or $j_{\beta}$. Take a $u_{\varepsilon}$ such that $\left[u_{\alpha}, u_{\varepsilon}\right] \neq 0$ and $\left[u_{\varepsilon}, u_{\beta}\right] \neq 0$. Then $\left[e_{i_{\alpha} j_{\alpha}}, e_{i_{\varepsilon}} j_{\xi}\right]=\delta_{j_{\alpha} i_{\xi}} e_{i_{\alpha} j_{\varepsilon}}-\delta_{j_{\varepsilon} i_{\alpha}} e_{j_{\varepsilon} i_{\alpha}} \neq 0$. Hence $i_{\varepsilon}=j_{\alpha}$ or $i_{\alpha}=j_{\varepsilon}$. It follows similarly that $i_{\S}=j_{\beta}$ or $j_{\varepsilon}=i_{\beta}$. Now there exists an element $h(\neq 0)$ of $\mathfrak{J y}_{\text {J }}$ such that $[h, \mathcal{H}]=0 . .^{19)}$ Then $\left[h, u_{\curlyvee}\right]=0$ for every $\boldsymbol{u}_{\curlyvee}$. Let $h=\lambda^{1} h_{1}+\ldots$ $+\lambda^{n+1} h_{n+1}$. It follows that $\lambda^{i_{\alpha}}=\lambda^{j_{\alpha}}, \lambda^{i_{\varepsilon}}=\lambda^{j_{\varepsilon}}$ and $\lambda^{i_{\beta}}=\lambda^{j_{\beta}}$. From the above relations we obtain $\lambda^{i_{\alpha}}=\lambda^{j_{\alpha}}=\lambda^{i_{\varepsilon}}=\lambda^{j_{\varepsilon}}=\lambda^{i_{\beta}}=\lambda^{j_{\beta}}$. Hence $\lambda^{s}=\lambda^{t}$. Since this holds for every pair of $s$ and $t$, it follows that $\lambda^{1}=\lambda^{2}=\ldots=\lambda^{n+1}$. Then necessarily $\lambda^{1}=\lambda^{2}=\ldots=\lambda^{n+1}=0$, since $\sum_{i=1}^{n+1} \lambda^{i}=0$. Hence $h=0$ and this is impossible. Thus $S \neq\{1,2, \ldots, n+1\}$. Hence there exists an integer $s(1 \leqq s$ $\leqq n+1)$ such that $s \bar{E} S$. Then we see easily that $\mathfrak{U}$ is contained in the Lie algebra $\mathfrak{A}=\mathfrak{h}^{\prime}+\sum_{\substack{i, k=1 \\ i \neq 1, k \neq s}}^{n+1} e_{i \hat{k}} K$, where $\mathfrak{h}^{\prime}$ is composed of all elements $\lambda^{1} h_{1}+\ldots$ $+\lambda^{n+1} h_{n+1}$ of $\mathscr{S}$ such that $\lambda^{s}=0$. But since $\mathfrak{U}$ and $\mathfrak{A}$ have the same dimension, it follows that $\mathfrak{U}=\mathfrak{A}$. Then every matrix of $\mathfrak{u}$ transforms the $s$-th axis of the $(n+1)$-dimensional complex vector space into 0 . Then "unitary ristricted" $\mathfrak{u}_{c}$ transforms the $s$ th axis of the unitary $(n+1)$-space into 0 . The integrated group $U$ leaves fixed the same axis. Then clearly $U$ is conjugate to $A_{n-1}$.

IV.

1. We consider now the group $G=C_{n}$, the unitary simplectic group of $2 n$ variables. $G$ consists of all unitary matrices of degree $2 n$ which leave the skewsymmetric bilinear form

$$
S(x, y)=\left(x_{1} y_{1}^{\prime}-x_{1}^{\prime} y_{1}\right)+\left(x_{2} y_{2}^{\prime}-x_{2}^{\prime} y_{2}\right)+\ldots+\left(x_{n} y_{n}^{\prime}-x_{n}^{\prime} y_{n}\right)
$$

invariant, where the vector $x$ has the componentes $\left(x_{1}, \ldots, x_{n}, x_{1}^{\prime}, \ldots, x_{n}{ }^{\prime}\right)$.

We denote by $C_{n-1}$ the subgroup of $G$ conisting of all matrices of $G$ which leave fixed the variables $x_{1}$ and $x_{1}^{\prime}$ i.e. $x_{1} \rightarrow x_{1}, x_{1}^{\prime} \rightarrow x_{1}^{\prime}$. Then $G / C_{n-1}=S^{4 n-1}$ and hence $C_{n-1}+0 . .^{12}$

We prove the following

Theorem III. Every S-subgroup of $G=C_{n}$ is conjugate to $C_{n-1}$ for $n>4$.

19) Take an element $h_{\mathfrak{1}}$ of $\mathfrak{g}$ which is not contained in $\mathfrak{y}$. Since $\left[h_{\mathfrak{1}}, \mathfrak{u}\right] \subseteq \mathfrak{u}$, the mapping $u \rightarrow\left[h_{1}, u\right](u \in \mathfrak{l})$ is a derivation of $\mathfrak{u}$. As the derivations of the simple Lie algebra $\mathfrak{u}$ are inner, there exists an element $u_{0}$ of $\mathfrak{u}$ such that $\left[h_{\mathfrak{1}}, u\right]=\left[u_{0}, u\right]$ for every $u \in \mathfrak{u}$. But since $\left[h_{1}, u\right]=0$ for every $u \in \mathfrak{h}, \boldsymbol{u}_{0}$ commutes with every element of $\mathfrak{h}$. $\mathfrak{h}$ is a maximal abelian subalgebra of $\mathfrak{u}$ and hence $\boldsymbol{u}_{0} \in \mathfrak{h}$. Then the element $h_{1}-\boldsymbol{u}_{0}$ satisfies our condition. 
2. Let $U$ bc an $S$-subgroup of $G$. Then $R(G / U)=R\left(S^{m}\right) \quad(m$ : odd) and $R(G)=R\left(U \times S^{m}\right)$. The homology ring of $G$ is

$$
R(G)=R\left(C_{n}\right)=R\left(S^{3} \times S^{7} \times \ldots \times S^{4 n-1}\right) !^{(5)}
$$

Hence $m=4 k-1$ and

$$
R(U)=R\left(S^{3} \times S^{7} \times \ldots \times S^{4 k-5} \times S^{4 k+3} \times \ldots \times S^{4 n-1}\right),
$$

Since $S^{3}$ appears in (1) only once, $U$ is simple. $0^{(5)}$ As we may easily verify, $U^{P}$ can not be the exceptional groups $F_{4} E_{3}$ and $E_{3}$. If $U=E_{6}$, then we may casily verify that the homology ring of $E_{6}$ must be isomorphic with that of $C_{6}$ and this is a contradiction. ${ }^{01}$ Hence if $n>3, U$ is a classical simple group. Then (1) is impossible if $n<4 n-1$. Hence $n=4 n-1$ and $R(U)=R\left(S^{3} \times S^{2} \times \ldots\right.$ $\left.\times S^{12-5}\right)$. This shows that $U$ is a simple group of type $C_{n-1}$ or $B_{n-11^{15}}$

3. Let the tie algebras $\mathfrak{B}_{c}, \mathfrak{B}, \mathfrak{U}_{c}, \mathfrak{H}_{,} \mathfrak{S}_{\mathfrak{c}}, \mathfrak{F}_{\mathfrak{g}}, \mathfrak{h}_{c}$ and $\mathfrak{h}$ be defined as in III. 3 , Then $B$ has the following basis:

$$
\mathscr{G}=K h_{1}+\ldots+K h_{n}+\sum_{i=1}^{n} K e_{ \pm 2 \lambda^{i}}+\sum_{i, k=1}^{n} K e_{ \pm \lambda^{i} \pm \lambda^{k}}
$$

( $K$ : the field of complex numbers),

where $\mathscr{S}=K h_{1}+\ldots+K h_{n}$ and $\left[\sum_{j=1}^{n} \lambda^{j} h_{j}, e_{ \pm 2 \lambda^{i}}\right]=\left( \pm 2 \lambda^{i}\right) e_{ \pm 2 \lambda^{i}}$ and $\left[\sum_{j=1}^{n} \lambda_{j} h_{j}\right.$ $\left.\boldsymbol{e}_{ \pm \lambda^{i} \pm \lambda^{k}}\right]=\left( \pm \lambda^{i} \pm \lambda^{k}\right) e_{ \pm \lambda^{i} \pm \lambda^{k}}$. First let $\mathfrak{U}$ be a simple algebra of type $C_{n-1}$. Then $\mathfrak{u}$ has the following basis :

$$
\mathfrak{u}=K \tilde{h}_{1}+\ldots+K \tilde{h}_{n-1}+\sum_{i=1}^{n-1} K u_{ \pm 2 \lambda^{i}}+\sum_{i, k=1}^{n-1} K u_{ \pm} \lambda^{i} \pm \lambda^{k},
$$

where $\mathfrak{h}=K \widetilde{h}_{1}+\ldots+K \tilde{h}_{n-1}(\subset \mathfrak{F})$ and the commutator products of $\tilde{h}$ 's and $u$ 's are defined anlogously as in $\mathbb{S}$. If $\mathfrak{l}$ is a simple algebra of type $B_{n-1}, \mathfrak{l}$ has the following basis:

$$
\mathfrak{u}=K \tilde{h}_{1}+\ldots+K \widetilde{h}_{n-1}+\sum_{i=1}^{n-1} K u_{ \pm \lambda^{i}}+\sum_{i, k=1}^{n-1} K u_{ \pm \lambda^{i} \pm \lambda^{k}}
$$

where $\mathfrak{h}=K \widetilde{h}_{1}+\ldots+K \tilde{h}_{n-1} \quad(\subset \mathscr{g})$ and $\left[\sum_{i=1}^{n-1} \lambda^{j} \widetilde{h}_{j}, u_{ \pm \lambda^{i}}\right]=\left( \pm \lambda^{i} u_{ \pm \lambda^{i}}\right)$ and $\left[\sum_{j=1}^{n-1} \lambda^{j} \tilde{h}_{j}, u_{ \pm} \lambda_{ \pm}^{i} \lambda^{k}\right]=\left( \pm \lambda^{i} \pm \lambda^{k}\right) u_{ \pm \lambda^{i} \pm \lambda^{k}}$. Clearly $u_{\alpha}$ 's are the linear combinations of $e_{\beta}^{\prime}$ 's. A $u_{\alpha}$ is said to be singular if it is a linear combination of at least two $\boldsymbol{e}_{\beta}$ 's. We shall prove that non of them is singular in case $n>4$ 。

20) See, Yen Chih-Ta, Sur les polynomes de Poincaré des groupes simples exceptionnels, C. R. Paris, 228, (1949). 
4. Let $\tilde{h}_{i}=\sum_{k=1}^{n} \mu_{i}^{k} h_{k}$. Since $\tilde{h}_{i}$ are linearly independent the matrix $\left(\mu_{i}^{k}\right)$ $(k=1, \ldots, n ; i=1, \ldots, n-1)$ has rank $n-1$. Let $\mu^{k}=\left(\mu_{1}^{k}, \ldots, \mu_{n-1}^{k}\right)$, $k=1, \ldots, n$. Then $n-1$ of these $n$ vectors are linearly independent. We may assume that $\mu^{1}, \mu^{2}, \ldots, \mu^{n-1}$ are so. Now

$$
\left[\sum_{j=1}^{n-1} \lambda^{j} \tilde{h}_{j}, e_{\varepsilon \lambda^{i}+\delta \lambda^{k}}\right]=\left(\sum_{j=1}^{n-1} \lambda^{j}\left(\varepsilon \mu_{j}^{i}+\delta \mu_{j}^{k}\right)\right) e_{\varepsilon \lambda^{i}+\delta \lambda^{k}},
$$

where $\varepsilon= \pm 1, \delta= \pm 1$ for $i \neq k$ and for $i=k, \varepsilon=\delta= \pm 1$, if $\mathfrak{u}$ is of type $C_{n-1}$, and $\varepsilon=0, \delta= \pm 1$, if $\mathfrak{u}$ is of type $B_{n-1}$.

If $\sum_{j=1}^{n-1} \lambda^{j}\left(\varepsilon \mu_{j}^{i}+\delta \mu_{j}{ }^{k}\right)=\sum_{j=1}^{n-1} \lambda^{j}\left(\varepsilon^{\prime} \mu_{j}^{r}+\delta^{\prime} \mu_{j}^{s}\right)$ holds for every $\lambda^{j}$, then the relation $\varepsilon \mu^{i}+\delta \mu^{k}=\varepsilon^{\prime} \mu^{r}+\delta^{\prime} \mu^{s}$ holds. In this case we write $e_{\varepsilon \lambda^{i}+\delta \lambda^{k}} \equiv e_{\varepsilon^{\prime} \lambda} \lambda^{r}+\delta^{\prime} \lambda^{s}$. If $e_{\alpha}$ and $e_{\beta}$ appear in a singular $u_{\tau}$, then $e_{\alpha} \equiv e_{\beta}$.

5. Let $\mu^{n}=0$. Then the possible relations are $e_{\varepsilon \lambda^{i}+\lambda^{i c}} \equiv e_{\varepsilon \lambda^{i}-\lambda^{n}}(\varepsilon= \pm 1$; $1 \leqq i \leqq n)$. Hence the possible singular $u$ 's are the forms

$$
u_{\alpha_{i}}=a_{i} e_{\lambda^{i}+\lambda^{n}}+b_{i} e_{\lambda^{i}-\lambda^{n}}, u_{-\alpha_{i}}=c_{i} e_{-\lambda^{i}-\lambda^{n}}+d_{i} e_{-\lambda^{i}+\lambda^{n}}
$$

Then $\left[u_{\alpha_{i}}, u_{-\alpha_{i}}\right]=h+b_{i} c_{i} N_{i} e_{-2 \lambda^{n}}+a_{i} d_{i} N_{i}^{\prime} e_{2 \lambda^{n}} \in \mathfrak{h}$, where $h \in \mathfrak{h}$ and $\left[e_{\lambda^{i}-\lambda^{n}}\right.$, $\left.e_{-\lambda^{i}-\lambda^{n}}\right]=N_{i} e_{-2 \lambda^{n}}$ and $\left[e_{\lambda^{i}+\lambda^{n}}, e_{-\lambda^{i}+\lambda^{n}}\right]=N_{i}^{\prime} e_{2 \lambda^{n}}$ and $N_{i} \neq 0, N_{i}^{\prime} \neq 0$. Hence it follows $b_{i} c_{i}=a_{i} d_{i}=0$. This shows that $u_{\alpha_{i}}$ and $u_{-\alpha_{i}}$ can not be singular.

6. Now let $\mu^{n} \neq 0$. Then $\mu^{n}=\sum_{i=1}^{n-1} a_{i} \mu^{i}$. Let $m$ be the number of indices $i$

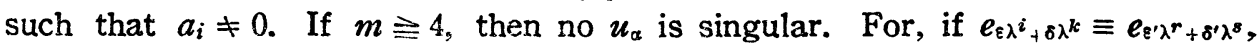
then $\varepsilon \mu^{i}+\delta \mu^{k}=\varepsilon^{\prime} \mu^{r}+\delta^{\prime} \mu^{s}$ and at least one of these vectors must be $\mu^{n}$. Then $\mu^{n}$ is a linear combination of at most 3 vectors and this impossible. Hence we may assume $m \leqq 3$.

i) First let $m=1$. For simplicity let $\mu^{n}=a_{3} \mu^{1}$. Let $\alpha=\varepsilon \lambda^{i}+\delta \lambda^{k}$. We denote for simplicity the indices $i, k$ as the indices of $\alpha$ and $e_{\alpha}$. Let $u_{\alpha}=\sum_{i} a_{\beta i} e_{\beta i}$ be singular. We show that the indices of $\beta_{i}$ 's are 1 and $n$. Suppose that $\beta_{3}$ has an index $i$ different from 1 and $n$ and let $\beta_{1}=\varepsilon_{1} \lambda^{i}+\varepsilon_{2} \lambda^{j}$. Further let $\beta_{2}=\eta_{1} \lambda^{k}+\eta_{2} \lambda^{l}$. Then since $e_{\beta 1} \equiv e_{\beta 2}, \varepsilon_{1} \mu^{i}+\varepsilon_{2} \mu^{j}=\eta_{1} \mu^{k}+\eta_{2} \mu^{l}$. If $i, j, k, l<n$, this is impossible. Since $i \neq 1, n, \alpha)$ first let $j=n$. Then $\varepsilon_{1} \mu^{i}+\varepsilon_{2} a_{1} \mu^{1}=\eta_{1} \mu^{k}$ $+\eta_{2} \mu^{l}$. Since $i \neq 1$, it follows that $i=k, \varepsilon_{1}=\eta_{1}$ and $1=l, \varepsilon_{2} a_{1}=\eta_{2}$. Then $\beta_{1}$ $=\varepsilon_{1} \lambda^{i}+\varepsilon_{2} \lambda^{n}$ and $\beta_{2}=\varepsilon_{1} \lambda^{i}+\varepsilon_{2} a_{1} \lambda^{1}$. $\beta$ ) Let $k=n$. Then $\beta_{1}=\varepsilon_{1} \lambda^{i}+\varepsilon_{2} \lambda^{1}$ and $\beta_{2}$ $=\varepsilon_{1} \lambda^{i}+\varepsilon_{2} a_{1} \lambda^{n}$. $\gamma$ ) Let $l=n$. Then $\beta_{1}=\varepsilon_{1} \lambda^{i}+\varepsilon_{2} \lambda^{3}$ and $\beta_{2}=\varepsilon_{1} \lambda^{i}+\varepsilon_{2} a_{1} \lambda^{n}$. In either case $\beta_{2}$ is determined by $\beta_{1}$ uniquely, whence $e_{\beta 3}$ can not appear in $u_{\alpha}$,

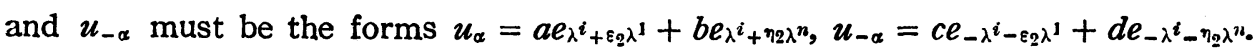
Then $\left[u_{\alpha}, u_{-\alpha}\right] \in \mathfrak{h}$ and we may prove in the same way as in 5 that $b c=0$ and $a d=0$. Then $u_{\alpha}$ and $u_{-\alpha}$ are not singular. Hence the indices of $\beta_{i}$ are 1 


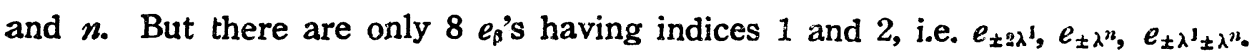
Hence the number of possible singular $u$ 's is at most 4 .

ii) Next let $m=2$. For simplicity let $\mu^{n}=a_{1} \mu^{1}+a_{2} \mu^{2}$. If $u_{\alpha}=\sum_{i} a_{\beta i} e_{\beta i}$ is singular, then we may prove as in i) that the indices of $\beta_{i}$ 's form subset of $\{1,2, n\}$. But in each singular $u_{a}$ at least one $e_{\beta i}$ must appear, one of whose

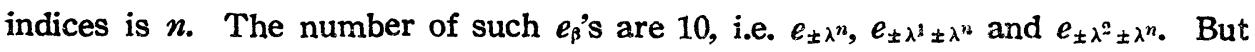
it is impossible that, for example, $e_{\lambda^{1}+\varepsilon \lambda^{n}}$ and $e_{-\lambda^{1}+\varepsilon \lambda^{n}}$ both appear in the singular $u$ 's. For, then the relations $e_{\varepsilon_{1} \lambda^{1}+\varepsilon_{2} \lambda^{2}+\varepsilon_{3} \lambda^{3}} \equiv e_{\lambda^{1}+\varepsilon \lambda^{n}}$ and $e_{\delta,} \lambda^{1}+\delta 2 \lambda^{2}+\delta_{3} \lambda^{n} \equiv e_{-\lambda^{1}+\varepsilon \lambda^{n}}$ hold, where $\varepsilon_{i}, \delta_{i}= \pm 1$ or 0 and at least one of $\varepsilon_{i}$ and $\delta_{i}$ is 0 respectively. These lead to a contradiction as we may easily verify. From these facts we see that the number of the possible singular $u$ 's is at most 6 .

iii) Finally let $m=3$ and let, for simplicity, $\mu^{n}=a_{1} \mu^{1}+a_{2} \mu^{2}+a_{3} \mu^{3}$. Then as above the singular $u$ 's are the linear combinations of $e_{\beta i}$ 's whose indices form subsets of $\{1,2,3, n\}$ and in each $u_{\alpha}$ at least one $e_{\beta_{i}}$ with index $n$ must appear.

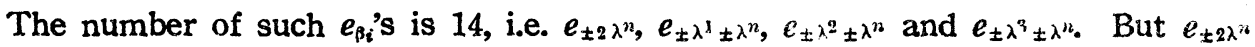
can not appear in the singular $u$ s, for if did $\mu^{n}$ would be a linear combination of two $\mu^{\prime} s$. As in ii) it is also impossible that, for example, $e_{\lambda^{1}+\varepsilon \lambda^{n}}$ and $e_{-\lambda^{1}+\varepsilon \lambda^{\prime \prime}}$ both appear in the singular $u$ 's. Hence the number of possible singular $u$ 's is at most 6. Thus we have shown that the number of singular elements is at most 6 . We see also from the above consideration that if the number $s$ of singular elements is 6 , then for every singular $u_{\beta}, u_{-\beta}$ is also singular. Further we see that if $s=5$, then there exist singular $u_{\beta_{1}}$ and $u_{\beta_{2}}$ such that $u_{-\beta_{3}}$ and $u_{-\beta_{2}}$ are also singular. These hold equally for $\mathfrak{u}$ of type $B_{n_{-1}}$ and of type $C_{n-1}$.

7. Next we prove that if $n>4$, no $u$ is singular.

i) Let $\mathfrak{u}$ be the type $B_{n-\jmath}$. Suppose that $u_{\varepsilon \lambda^{i}+\delta \lambda^{k}}(i \neq k, \varepsilon= \pm 1, \delta= \pm 1)$ is singular. Then since $\left[u_{\varepsilon \lambda^{i}}, u_{\delta \lambda^{k}}\right]=N_{i, k} u_{\varepsilon \lambda^{i}+\delta \lambda^{k}}, u_{\xi \lambda^{i}}$ or $u_{\delta \lambda^{k}}$ must be singular. Let $u_{\varepsilon \lambda^{i}}$ be singular. Then since $u_{\xi \lambda^{i}}=N_{t}\left[u_{\varepsilon \lambda^{i}+\lambda^{t}}, u_{-\lambda}\right],(t=1, \ldots, n-1$, $t \neq i), u_{\varepsilon \lambda^{i}+\lambda^{t}}$ or $u_{-\lambda^{t}}$ is singular for every $t$. Hence we get a set of $n-2$ singular elements. Further from the relations $u_{\varepsilon \lambda^{i}}=N_{t}^{\prime}\left[u_{\varepsilon \lambda^{i}-\lambda^{t}}, u_{\lambda} t\right](t=1, \ldots$, $n-1, t \neq i$ ), we get also a set of $n-2$ singular elements and these two sets have no common elements. $u_{\lambda \varepsilon^{i}}$ is also a singular element different from these $2(n-2)$ singular element. Hence there are at least $2(n-2)+1$ singular elements. If the number $s$ of singular elements is $\leqq 4$, then $2(n-2)+1 \leqq 4$ and hence $n \leqq 3$. If $s=5$ we see easily that there exists at least one singular $u_{3}$ different from the above $2(n-2)+1$ elements and hence also $n \leqq 3$. If $s=6$, there exist at least two singular elements different from the above $2(n-2)+1$ element and so also $n \leqq 3$. Hence we may assume that all $u_{\alpha}\left(\alpha= \pm \lambda^{i} \pm \lambda^{k}, i\right.$, $k=1, \ldots, n-1)$ are not singular. If one of $u_{ \pm} \lambda^{i}$, say $u_{\lambda^{1}}$, is not singular, 
then since $\left[u_{\lambda^{1}}, u_{-\lambda^{1} \pm \lambda^{i}}\right]=N_{i} u_{ \pm \lambda^{i}}(i=2, \ldots, n=1), u_{ \pm \lambda^{i}}(i=2, \ldots, n-1)$

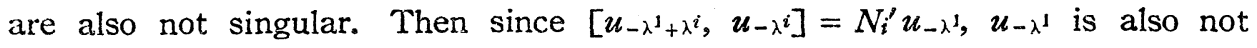
singular and hence all $u$ 's are not singular. If all of $u_{ \pm \lambda^{i}}(i=1, \ldots, n-1)$ are singular, there exist $2(n-1)$ singular element. Hence $2(n-1) \leqq 6$, i.e. $n \leqq 4 .{ }^{21}$ ) Thus if $n>4 u$ s are not singular.

ii) If $\mathfrak{u}$ is of type $C_{n_{-1}}$, we may prove analogously that none of $u$ is singular in case $n>3$.

8. We prove now that $u$ is not of type $B_{n-1}$. Let $u_{\lambda^{i}}=a_{i} e_{\alpha_{i}}$ and $u_{-\lambda^{i}}$ $=b_{i} e_{-\alpha_{i}}(i=1,2, \ldots, n-1)$. Then since $\left[u_{\varepsilon \lambda^{i}}, u_{\delta \lambda^{j}}\right] \neq 0(\varepsilon, \delta= \pm 1)$, we see that the set of $2(n-1)$ "roots" $\left\{ \pm \alpha_{i}\right\}$ of $\&$ has the property that $\pm \alpha_{i} \pm \alpha_{j}$ are also the roots of $\Theta$. Let one of $\alpha_{i}$ be the form $\pm 2 \lambda^{j}$; for example let $\alpha_{1}=2 \lambda^{j}$. Further let $\alpha_{2}=\varepsilon \lambda^{i}+\delta \lambda^{k}$, then since $\alpha_{1}+\alpha_{2}$ is a root, it follows that $\alpha_{2}=-\lambda^{j}+\delta \lambda^{k}$. But then $-\alpha_{1}+\alpha_{2}=-2 \lambda^{j}-\lambda^{j}+\delta \lambda^{k}$ is not a root of $\mathbb{G}$. Hence $\alpha_{i}$ are of the forms $\pm \lambda^{k} \pm \lambda^{j}$. Let $\alpha_{1}=\varepsilon \lambda^{i}+\delta \lambda^{k}$ and $\alpha_{2}=\varepsilon_{1} \lambda^{j}+\delta_{1} \lambda^{l}$. Since $\alpha_{1}+\alpha_{2}=\varepsilon \lambda^{i}+\delta \lambda^{k}+\varepsilon_{1} \lambda^{j}+\delta_{1} \lambda^{l}$ is a root, it follows that $\alpha_{2}=-\varepsilon \lambda^{i}+\eta \lambda^{s}$ or $\alpha_{2}=-\delta \lambda^{k}+\eta \lambda^{s}$, where $s=j$ or $l$. But since $\alpha_{1}-\alpha_{2}$ is also a root it follows that $\alpha_{2}=-\varepsilon \lambda^{i}+\delta \lambda^{k}$ or $\alpha_{2}=\varepsilon \lambda^{i}-\delta \lambda^{k}$. Thus $\alpha_{2}$ and $-\alpha_{2}$ are determined uniquely by $\alpha_{1}$. Hence if $n>3$, there is no such a set of roots $\left\{ \pm \alpha_{i}\right\}$ of $\&$. Therefore Il is not of type $B_{n-1}$,

9. By 8. we know that $\mathfrak{u}$ is of type $\dot{C}_{n-1}$. Now let $u_{2 \lambda} i=a_{i} e_{\alpha i}$ and $u_{-2 \lambda} i$ $=b_{i} e_{-\alpha_{i}}(i=1,2, \ldots, n-1)$. Since $\left[u_{\varepsilon 2 \lambda^{i}}, u_{\delta 2 \lambda^{j}}\right]=0$, the set of $2(n-1)$ roots $\left\{ \pm \alpha_{i}\right\}$ of $\mathbb{S}$ has the property that $\pm \alpha_{i} \pm \alpha_{j}$ are not the roots of $\mathbb{B}$ at all. Suppose that $\alpha_{j}=\varepsilon_{j} 2 \lambda^{i_{j}}$ for $1 \leqq j \leqq k$ and $\alpha_{s}=\delta_{s} \lambda^{j_{s}}+\eta_{s} \lambda^{l_{s}}\left(j_{s} \neq l_{s}\right)$ for $k+1$ $\leqq s \leqq n-1$. Then the sets of indices $\left\{i_{1}\right\}, \ldots,\left\{i_{k}\right\},\left\{j_{k+1}, l_{k+1}\right\}, \ldots,\left\{j_{n-1}\right.$, $\left.l_{n-1}\right\}$ have no common indices. Hence the number of these indices is $k+2(n$ $-k-1)=2(n-1)-k$. Thus $2(n-1)-k \leqq n$ and hence $k \geqq n-2$. Suppose that $k=n-2$ and let, for simplicity, $\left\{i_{1}, \ldots, i_{n-1}\right\}=\{1,2, \ldots, n-2\},\left\{j_{n-1}\right.$, $\left.l_{n-1}\right\}=\{n-1, n\}$ and $u_{2 \lambda^{n}}=a_{n-1} e_{2 \lambda^{n-1}+\delta \lambda^{n}}$. Now there exists an element $h=\lambda^{1} h_{1}$ $+\ldots+\lambda^{n} h_{n} \in \mathscr{S}(h \neq 0)$ such that $[h, u]=0$ for all $u \in \mathfrak{H}^{19)}$ Since $\left[h, u_{2 \lambda^{i}}\right]$ $=0$, it follows that $\lambda^{1}=\lambda^{2}=\ldots=\lambda^{n-2}=0$ and $\varepsilon \lambda^{n-1}+\delta \lambda^{n}=0$. Let $u_{\lambda^{n-1}+\lambda^{i}}$ $=c e_{\beta 1}$ and $u_{\lambda^{n-1}-\lambda^{i}}=d e_{\beta 2}$. As $\left[h, e_{\beta k}\right]=0 \quad(k=1,2)$ and $\beta_{k} \neq \pm\left(\varepsilon \lambda^{n-1}+\delta \lambda^{n}\right)$,

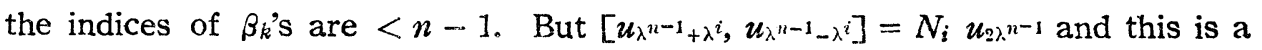
contradiction. Hence $k=n-1$. Suppose $\left\{i_{1}, \ldots, i_{n-1}\right\}=\{1,2, \ldots, n-1\}$. Then we easily see that $u_{\varepsilon \lambda^{i}+\delta \lambda^{k}}=a_{i k} e_{\varepsilon j \lambda^{j}+\delta_{1} \lambda^{l}}(1 \leqq i, k \leqq n-1)$ and $1 \leqq j, l \leqq n-1$. Now let $\mathfrak{A}=K h_{1}+\ldots+K h_{n+1}+\sum_{i=1}^{n-1} K e_{ \pm 2 \lambda^{i}}+\sum_{i, k=1}^{n-1} K e_{ \pm \lambda^{i} \pm \lambda^{k}}$. Then $\mathfrak{U}$ is a subalgebra of $\mathfrak{B S}$ of type $C_{n-1}$ containing $\mathfrak{u}$. Hence $\mathfrak{A}=\mathfrak{u}$. Then each matrix of $\mathfrak{u}$

21) If $n=4$, there may be 6 singular elements. 
transforms the variables $x_{n}$ and $x_{n}^{\prime}$ into 0 . The same holds for every matrix of $\mathfrak{u}_{c}$. Hence each matrix of the integrated group $U$ leaves fixed the variables $x_{n}$ and $x_{n}^{\prime}$ (i.e. $x_{n} \rightarrow x_{n}, x_{n}{ }^{\prime} \rightarrow x_{n}{ }^{\prime}$ ). Then clearly $U$ is conjugate to $C_{n-1}$. Thus Theorem III is proved.

V.

Now let $G$ be the exceptional group of rank $>2$. The Poincare polynomials of the exceptional groups are as follows: ${ }^{20}$

$G_{2}:\left(1+x^{3}\right)\left(1+x^{11}\right)$;

$F_{4}:\left(1+x^{3}\right)\left(1+x^{31}\right)\left(1+x^{15}\right)\left(1+x^{93}\right) ;$

$E_{6}:\left(1+x^{3}\right)\left(1+x^{9}\right)\left(1+x^{11}\right)\left(1+x^{15}\right)\left(1+x^{17}\right)\left(1+x^{23}\right) ;$

$E_{7}:\left(1+x^{3}\right)\left(1+x^{11}\right)\left(1+x^{15}\right)\left(1+x^{19}\right)\left(1+x^{23}\right)\left(1+x^{27}\right)\left(1+x^{35}\right) ;$

$E_{9}:\left(1+x^{3}\right)\left(1+x^{15}\right)\left(1+x^{23}\right)\left(1+x^{27}\right)\left(1+x^{35}\right)\left(1+x^{39}\right)\left(1+x^{47}\right)\left(1+x^{59}\right)$.

From this table, we may easily see the following

THEOREM IV. Exceptional simple groups of rank $>2$ have no S-subgroups.

Mathematical Institute, Nagoya University. 